\title{
Integrated Optimization of Flexible Job Shop Scheduling Problem and Equipment Maintenance Plan
}

\author{
Zha Jing $^{1, a}$,Jin Hua ${ }^{2, b}$, Pan Zhicheng ${ }^{3, c}$ and Yuan Qingsong ${ }^{4, d}$ \\ 1,2,3,4 College of Mechanical \& Power Engineering of China \\ Three Gorges University, Hubei Yichang, 443002, China \\ bjinhua_vds@163.com
}

\begin{abstract}
Keywords: Flexible job shop; Shop Scheduling; Equipment Maintenance;
Abstract. This paper researches the integrated optimization of flexible job shop scheduling problem (FJSP) and equipment maintenance plan. The object of the integrated optimization model is to pursue the minimum of makespan. The paper proposed a two-stage algorithm based on the genetic algorithm to solve the problem. Firstly generated basic FJSP schedules. Secondly when the reliability of the equipment is below the threshold value probabilistically arranged the unequal periodic equipment maintenance at the equipment's free time. Then obtained an integrated plan. The optimization result of a practical example showed that the makespan of the integrated optimization was less than the makespan of equipment maintenance at regular intervals.
\end{abstract}

This work is supported by Science and technology research project from Hubei Provincial Department of Education (No. Q20151206)

\section{Introduction}

Flexible job-shop scheduling problem proved to be a NP-hard problem, and it's more complex than general Job-shop scheduling problem. Numerous researchers raised many algorithms to settle that problem efficiently. Wang, L[1](2012) proposed an improved artificial colony algorithm and Deming, L[2] (2013) presented a variable neighborhood search to solve FJSP. Most of FJSP study assumed that machines are available during whole scheduling process, which means the machines are never out of order. Actually equipment will produce fatigue or wear, performance reduction or even failure after running for some time. Therefore preventive maintenance is needed to prolong service life, ensure production run smoothly. Enterprises are often drawing up fixed time interval preventive maintenance according to production scheduling, such as after every 5 working days shutdown equipments for maintenance. Although this method is simple, it doesn't reflect the real operating conditions of equipment.

In the most of the existing studies, production scheduling and equipment maintenance are always applied separately. However, in the actual manufacturing system, they are closely interdependent (Berrichi[3] et al., 2008). On the one hand, equipment maintenance will occupy the production time, so that the performance of the scheduling is difficult to achieve the best. On the other hand, the production scheduling is highly dependent on the equipment. Once the equipment malfunctions, the entire production will be interrupted. Thus, it is necessary to investigates joint optimization of FJSP and Equipment Maintenance Plan. In the last few recent researches[4-6], the integrated optimization of production scheduling problem and preventive maintenance has been studied.

In this paper, optimization model for integrated FJSP and equipment maintenance plan has been established. Minimum completion time is considered as the objective. The time to failure of a machine is subject to Weibull distribution. Two-stage genetic optimization algorithm is proposed to resolve the problem. Computational result from instance elucidated that integrated optimization scheduling can not only guarantee continuous production, but also enhance production efficiency.

The rest of the paper is organized as follows. Section 2 gives detailed description of integrated problem. Section 3 describes the integrated mathematical model. Two-stage genetic optimization algorithm is discussed emphatically in Section 4. Section 5 presents the test problems and obtained results. Finally conclusions and future study are outlined in Section 6. 


\section{The integrated problem description}

The FJSP can be defined briefly as follows. There are collections of $m$ unrelated machines and $n$ jobs, every job concludes k different operations. The flexibility of the system is mainly given by the routing flexibility. Each job can be processed on some different devices with different processing time. In the scheduling, two decisions have to be made: (1) assigning jobs on machines and (2) determining the best working-starting time and sequence of jobs on each machine[7]. The goal is to make scheduling performance reach optimal. When it comes to maintenance, the starting and completing time of a production job will alter. According to this, FJSP and PM should be considered at the same time.

As the devises in processing workshop are mostly mechanical and electrical equipments, which failure rate density is closer to Weibull distribution (Cassady and Kutanoglu [8] (2005)). This article choose Weibull distribution to describe the equipment failure rate density, its function is given as Eq. (1)

$$
f(t)=\frac{\beta}{\eta}\left(\frac{t}{\eta}\right)^{\beta-1} \exp \left[-\left(\frac{t}{\eta}\right)^{\beta}\right], t>0, \beta>0, \eta>0
$$

Among them, $t$ is the running time of machines, $\beta$ is the shape parameter which decides curve shape of distribution. $\eta$ is scale parameter. Neither of them has something to do with. Then the expression of reliability function $R(t)$ at time $t$ is given as Eq.2.

$$
R(t)=1-\int_{t}^{\infty} f(t) \mathrm{dt}=\exp \left(-\left(\frac{t}{\eta}\right)^{\beta}\right)
$$

This paper manipulates an unequal periodic equipment maintenance strategy. After maintenance, reliability of equipments returns to initial value, while the reliability threshold will increase at the next time[9]. We assume all machines have same initial reliability threshold $R_{l, \text { min }}^{0}(e=0)$. Supposed that $e$ is the time of maintenance and $\alpha_{l}$ is the scaling factor. The increase of reliability threshold can describe as $R_{l, \min }^{e}=\left(1+b_{l}\right) R_{l, \min }^{e-1}$. The bigger $\alpha_{l}$ leads to faster increase of reliability threshold and shorter intervals of equipment maintenance.

\section{Mathematical model of integrated F JSP and PM}

Mathematical model proposed in this paper may be denoted as a development of the FJSP model proposed by Gao, Gen, and Sun[10] (2006). The main difference being represented by its adaptation to the FJSP and PM issue. The mathematical model can be expressed as follows, and the notations are defined as shown in Table 1.

Table.1. The notations

\begin{tabular}{l|l|}
\hline$i, h$ & Index of jobs $i, h=(1,2, \cdots, n)$ \\
\hline$j, d$ & Index of operation of jobs \\
\hline$l$ & Index of machines $l=(1,2, \cdots, m)$ \\
\hline$p_{(j, l}$ & The processing time of jobi of operation $j$ in machine $l$ \\
\hline$s t_{i j}$ & The starting time of jobi of operation $j$ \\
\hline$e t_{i j}$ & The completing time of jobi of operation $j$ \\
\hline$t_{l}$ & The maintenance time of machine $l$ \\
\hline$C_{i}$ & The completing time of jobi \\
\hline$C_{\max }$ & The max completing time \\
\hline$L$ & An arbitrary large positive number \\
\hline
\end{tabular}

Decision variables:

a. $x_{i j l}=1$ if the operation $j$ of job $i$ is processing on machine $l$, otherwise $x_{i j l}=0$. 
b. $g_{(i j)(h d) l}=1$ if the operation $j$ of job $i$ is processing before the operation $h$ of job $d$ on machine $l$, otherwise $g_{(i j)(h d) l}=0$.

c. $q_{(i j)(h d) l}=1$ if there is a maintenance between the operation $j$ of jobi and the operation $h$ of job $d$ on machine $l$, otherwise $q_{(i j)(h d) l}=0$

$$
\begin{aligned}
& \min \quad C_{\max }=\underset{i=1}{\min \left(\max _{i}\right)} \\
& \text { s.t. } \quad s t_{i j}+x_{(i j) l} \times p_{(i j) l} \leq e t_{i j} \\
& e t_{i j} \leq s t_{i(j+1)} \\
& e t_{i j} \leq C_{\max } \\
& \sum_{l=1}^{m} x_{i j l}=1 \\
& e t_{i j} \times x_{(i j) l}+t_{l} \times q_{(i j)(h d) l} \times g_{(i j)(h d) l} \leq s t_{h d} \times x_{h d l}+L\left(1-g_{(i j)(h d) l}\right) \\
& \mathrm{R}_{l}(t) \leq R_{l, \min }
\end{aligned}
$$

Eq.3 indicates pursuit of the shortest total completion time. Inequities (4) and (5) ensure that the predefined operation sequence of all jobs would be considered. Inequities (6) ensure that each operation completion time may not exceed the total completion time. Eq.(7) states that just one machine can be selected. Inequities (8) ensure that there are not overlaps between operations and maintenance activities on each machine. Inequities (9) ensure that the reliability of the equipment should be less than its reliability threshold.

\section{Two-stage genetic optimization algorithm}

Due to the integrated optimization problem is NP - hard problem, very difficult to solve directly. In this article, we design a two-stage algorithm based on genetic optimization. The framework of algorithm is shown in Fig.1.

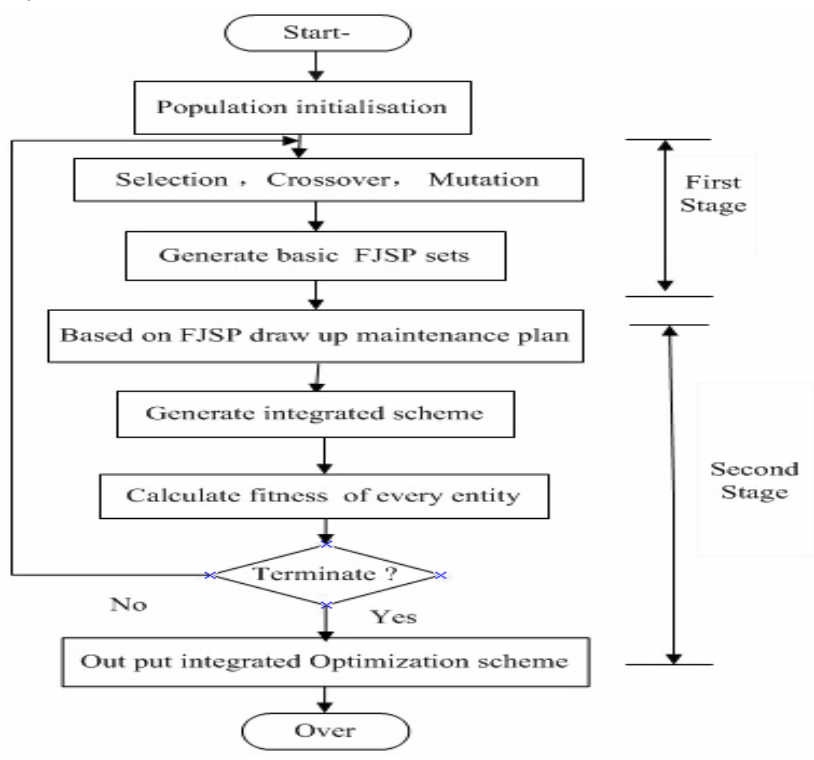

Fig.1. The framework of algorithm

Due to the conflicts between job processing and maintenance activities, there are two policies to insert maintenance activities. In the first strategy, until the operation finish maintenance will begin. On the contrary, the maintenance will insert before the operation start. They are called forward method and backward method. Fig.2 shows the instance adopted the two methods, dotted line is the moment when equipment reliability reached the threshold. To compare the two methods, we will discover that machine 2 use the idle time to maintain and the makespan is shorter. 


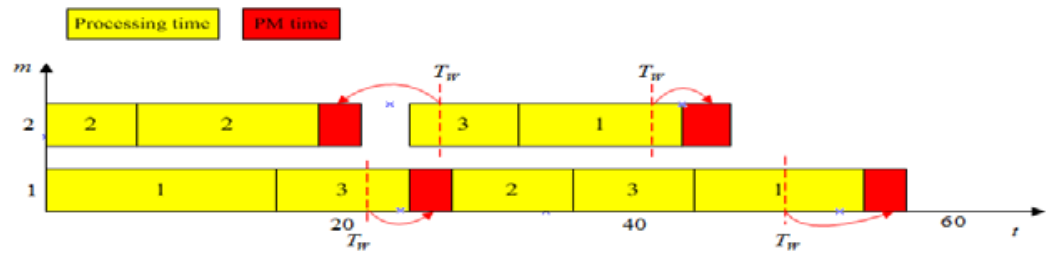

Fig.2. Forward and backward methods

In this paper a hybrid probability inserting of maintenance plan policy is proposed. Two indicators are designed: allowance coefficient of reliability $a_{l}$ and maintenance inserting probability $Y$. Its detailed procedure is described as follows:

Step 1 Select a basic FJSP scheduling, and make the current $O_{i j}$ the first one of operation order, then initialize the time of maintenance and initial reliability threshold.

Sept 2 Calculate the cumulative running time of machine at the moment of processing beginning and completing: $T_{i j l}$ and $\left(T_{i j l}+p_{i j l}\right)$, and corresponding reliability. If $R_{l}\left(T_{i j l}+p_{i j l}\right)>R_{l, \text { min }}^{e}$, indicates that there is no need for maintenance. Skip to step 4. If $R_{l}\left(T_{i j l}\right) \leq R_{l, \text { min }}^{e}$, insert a maintenance activities after the previous operation has been completed on machine $l$, and $e_{l}=e_{l}+1$. Ship to step 4 .

If $R_{l}\left(T_{i j l}\right)>R_{l, \min }^{e} \geq R_{l}\left(T_{i j l}+p_{i j l}\right)$ and $R_{l}\left(T_{i j l}+p_{i j l}\right)<a_{l} \times R_{l, \text { min }}^{e}$, insert a maintenance activities after the last operation has been finished on machine $l$, and $e_{l}=e_{l}+1$. Ship to step 4 . If and $R_{l}\left(T_{i j l}\right)>R_{l, \min }^{e} \geq R_{l}\left(T_{i j l}+p_{i j l}\right)$, skip to step 3 .

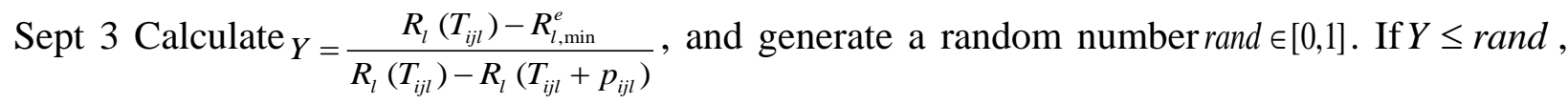
insert a maintenance activities after the last operation has been finished on machine $l$, update $e_{l}=e_{l}+1$. Skip to step 4 . If $Y>$ rand , first arrange processing of the current operation on machine $l$, and then insert a maintenance task. Update $e_{l}=e_{l}+1$, and skip to step 4.

Step 4 If the current operation is the last one of the operation order, count the makespan. Otherwise update the current operation with next one, skip to step 2.

\section{Test and Result}

The instance is a flexible job-shop, in which 6 jobs with 36 operations are to be performed on 6 machines. In the initial separate scheme, equipment maintenance is carried out every 30 time units. The processing time is shown in Table.2, and the time units for duration of maintenance activities are from the Table.3.

Table.2. Parameters of machines

\begin{tabular}{|c|c|c|c|c|c|c|c|c|c|c|c|c|c|c|c|c|c|c|}
\hline Jobs & \multicolumn{6}{|c|}{ Job 1} & \multicolumn{6}{|c|}{ Job 2} & \multicolumn{6}{|c|}{ Job 3} \\
\hline Operations & 1 & 2 & 3 & 4 & 5 & 6 & 1 & 2 & 3 & 4 & 5 & 6 & 1 & 2 & 3 & 4 & 5 & 6 \\
\hline Processing times of machine 1 & - & 16 & 15 & 8 & 11 & 2 & - & 10 & 16 & 1 & 20 & 12 & 8 & 3 & 9 & 7 & 14 & 4 \\
\hline Processing times of machine 2 & 8 & - & 16 & 15 & 11 & 20 & 6 & 5 & 6 & 14 & - & 5 & 9 & 9 & 11 & 10 & 3 & 14 \\
\hline Processing times of machine 3 & 12 & 15 & 3 & - & 8 & 17 & 9 & 2 & 6 & דיה & 16 & 7 & 18 & 11 & 18 & 7 & 14 & 9 \\
\hline Processing times of machine 4 & 13 & 7 & 8 & 8 & 18 & 10 & 15 & - & - & 2 & 15 & 16 & 2 & - & 10 & - & 5 & 10 \\
\hline Processing times of machine5 & 11 & 14 & - & 7 & -3 & 11 & 5 & 14 & 12 & 13 & 5 & - & 7 & 12 & - & 7 & 16 & 15 \\
\hline Processing times of machine6 & 9 & 11 & 5 & 15 & 10 & - & 8 & 4 & 15 & 5 & 8 & 10 & - & 12 & 9 & 8 & - & - \\
\hline Jobs & \multicolumn{6}{|c|}{ Job 4} & \multicolumn{6}{|c|}{ Job 5} & \multicolumn{6}{|c|}{ Job 6} \\
\hline Operations & 1 & 2 & 3 & 4 & 5 & 6 & 1 & 2 & 3 & 4 & 5 & 6 & 1 & 2 & 3 & 4 & 5 & 6 \\
\hline Processing times of machine 1 & 13 & 18 & 2 & 5 & 5 & 9 & 14 & 15 & 18 & 8 & 1 & 11 & 3 & 11 & 11 & 4 & 3 & - \\
\hline Processing times of machine 2 & 3 & 20 & 8 & - & 15 & 17 & - & - & 18 & - & 16 & 15 & 14 & 17 & 10 & 15 & 7 & 8 \\
\hline Processing times of machine 3 & 15 & 4 & 6 & 10 & - & 9 & 9 & 10 & 11 & 7 & 5 & 10 & 7 & 17 & 14 & 7 & 12 & 20 \\
\hline Processing times of machine 4 & 5 & 15 & 15 & 13 & 5 & 15 & 11 & 5 & 16 & 5 & 4 & 13 & - & 13 & 13 & - & 16 & 14 \\
\hline Processing times of machine5 & 5 & - & - & 17 & 4 & 18 & 4 & 14 & 9 & 12 & - & - & 14 & 7 & 10 & 7 & - & 3 \\
\hline Processing times of machine6 & - & 13 & 8 & 8 & 14 & - & 6 & 5 & - & 5 & 10 & 16 & 15 & - & דה- & 13 & 8 & 6 \\
\hline
\end{tabular}


Firstly, by adopting the basic GA, we obtained the initial scheme of FJSP and fixed time interval maintenance activities. Fig.3 shows the Gantt-chart for the initial separate scheme. The makespan is 138 time units and the number of maintenance activities is 19 times.

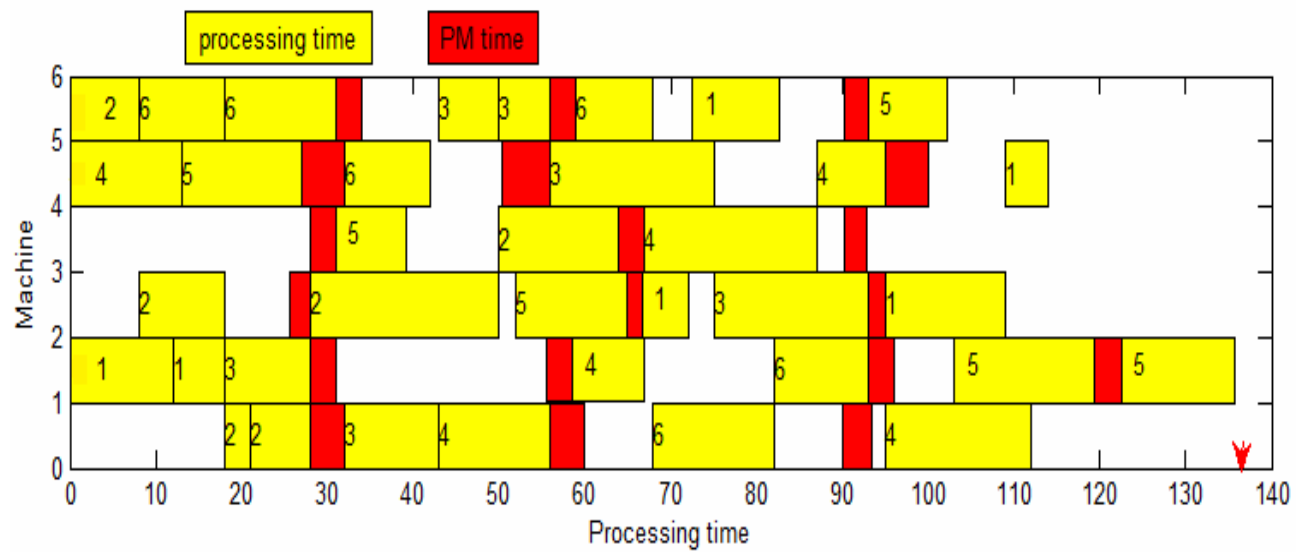

Fig.3. The initial separate scheme

Secondly, the two-stage genetic optimization algorithm described in Section 4 has been implemented. In our experiment, we tested different values for a list of algorithm parameters: population size is 100, maximal generation is 50, crossover probability is 0.8 and mutation probability is 0.06. Other parameters are depicted in table.3. The Gantt-chart for integrated optimization scheme of FJSP and equipment maintenance plan is shown in Fig.4.

Table.3. Operations' processing times on machines

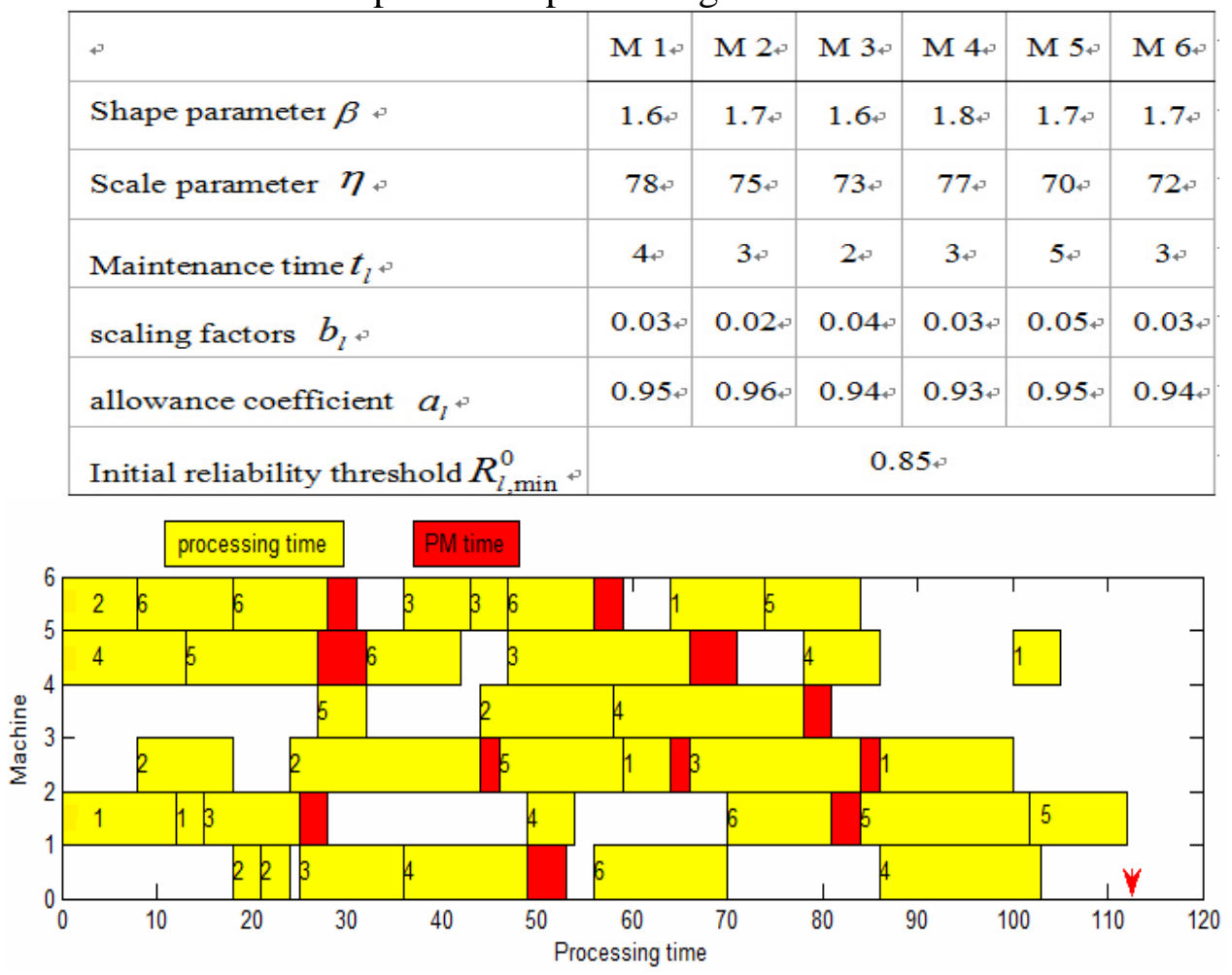

Fig.4.Gantt-chart for integrated scheme

The makespan of this scheme is 112 time units and the number of maintenance activities is 11 times. Now we compare the results obtained with the separated and integrated models. The results show that makespan reduced 26 time units and the number of maintenance activities decreased 8 times. Integrated optimization scheme get a $23.2 \%$ reduction in makespan. As a conclusion, integrated optimization scheme greatly improved the production efficiency and machine utilization. 


\section{Conclusion and further study}

In this paper, we developed an integrated model for FJSP and preventive maintenance, which aims to minimize makespan. Two-stage genetic algorithm has designed to solve the problem. In addition, the hybrid forward and backward methods have been applied to insert maintenance activities and use free time to maintenance. The integrated optimization of FJSP and equipment maintenance, improve the utilization rate of the machine and production efficiency.

For further studies, more sophisticated problem settings can be considered. Integrated Multi-targets, uncertain, and dynamic of FJSP and equipment maintenance would be deserved further investigation.

\section{References}

[1] Wang Ling, Zhou Gang, Xu Ye, Wang Sheng yao, Liu Min. An effective artificial bee colony algorithm for the flexible job-shop scheduling problem[J]. International Journal of Advanced Manufacturing Technology. 2012, 50(4): 303-315.

[2] Deming Lei, Xiuping Guo. Variable neighborhood search for dual-resource constrained flexible job shop scheduling. International Journal of Production Research. 2013, 52,(9): 2519-2529.

[3] Berrichi, A., Amodeo, L., Yalaoui, F., Châtelet, E., \& Mezghiche, M. (2008). Bi-objective optimization algorithms for joint production and maintenance scheduling. Application to the parallel machine problem. Journal of Intelligent Manufacturing, 20, 389-400.

[4] Khamseh A , Jolai F, Babaei M. Integrating sequence-dependent group scheduling problem and preventive maintenance in flexible flow shops[J]. International Journal of Advanced Manufacturing Technology. 2014, 77(4): 173-185.

[5] Naderi, B., M. Zandieh, and M. Aminnayeri. 2011. "Incorporating Periodic Preventive Maintenance into Flexible Flowshop Scheduling Problems.”Applied Soft Computing11 (2): 2094-2101.

[6] Cassady, C. R., and E. Kutanoglu. 2005. "Integrating Preventive Maintenance Planning and Production Scheduling for a Single Machine.” IEEE Transactions on Reliability 54 (2): 304-309.

[7] E. Moradi, S.M.T. Fatemi Ghomi, M. Zandieh. Bi-objective optimization research on integrated fixed time interval preventive maintenance and production for scheduling flexible job-shop problem. Expert Systems with Applications. 2011, 38, 7169-7178.

[8] Cassady, C. R., and E. Kutanoglu. 2003. "Minimizing Job Tardiness Using Integrated Preventive Maintenance Planning and Production Scheduling.” IIE Transactions 35 (6): 503-513.

[9] Shijin Wang. Bi-objective optimisation for integrated scheduling of single machine with setup times and preventive maintenance planning. International Journal of Production Research, 2013, 51, (12), 3719-3733.

[10] Gao, J., Gen, M., \& Sun, L. (2006). Scheduling jobs and maintenances in flexible job shop with a hybrid genetic algorithm. Journal of Intelligent Manufacturing, 17,493-507. 